\title{
Cooperative Antenna Selection Method for Directional Antenna Ad Hoc Networks Based on ALOHA
}

\author{
Bowen Zheng, ${ }^{1}$ Songlin Sun $\mathbb{D}^{1}{ }^{1}$ and Guoyuan Shao ${ }^{2}$ \\ ${ }^{1}$ School of Information and Communication Engineering, Beijing University of Posts and Telecommunications, Beijing 100000, China \\ ${ }^{2}$ North Automatic Control Technology Institute, Taiyuan 030006, China \\ Correspondence should be addressed to Songlin Sun; slsun@bupt.edu.cn
}

Received 8 February 2021; Revised 28 February 2021; Accepted 13 March 2021; Published 31 March 2021

Academic Editor: Xin Liu

Copyright (C) 2021 Bowen Zheng et al. This is an open access article distributed under the Creative Commons Attribution License, which permits unrestricted use, distribution, and reproduction in any medium, provided the original work is properly cited.

\begin{abstract}
In recent years, directional antennas or phased array antennas are being widely used in communication systems due to the higher antenna gains. However, without external time synchronization and angle synchronization, the unsynchronized node usually takes a long time to synchronize with the existing nodes due to the narrow beams. Although the multibeam transmission or the digital phased array antenna can reduce this problem, it is clear that the cost of the digital phased array antenna is currently too high. Without external time synchronization and angle synchronization, a cooperative antenna selection method based on directional antennas is proposed in this paper. Our method only uses the narrow beams to transmit and to receive and reduces the time for self-synchronization. In this paper, we give the expression of the expected average time for the self-synchronization of multiple nodes, transform the problem into the problem of finding the minimum value of the infinite norm of the sequence, and then propose a cooperative antenna selection method which calculates the optimal transmission probability distribution of the node in different directions through parameter sharing and relative geometric position relationship between nodes. Finally, we verify the proposed method through simulation, and the number of beams is set between 6 and 10. In a typical scenario of five nodes, our method reduces the maximum average self-synchronization time by $50 \%$ averagely, compared with the traditional method which sends the different antenna beams at equal probability.
\end{abstract}

\section{Introduction}

With the rapid growth of users' demand for big data services [1], the network throughput required is getting higher and higher. In order to improve spectrum utilization, many scholars have focused on cognitive radio [2], NOMA [2,3], etc. A simpler and more effective method is to use a higher frequency band, such as $5 \mathrm{G}$. A higher frequency band brings greater attenuation, so more communication systems use directional antennas which can increase transmission distance, reduce power consumption, and increase network capacity. However, the use of directional antennas brings new challenges to the MAC layer protocol design; the most critical one is how to coordinate and make full use of multiple antenna beam resources to achieve self-synchronization and dynamic neighbor discovery of the communication nodes.
Neighbor discovery is the precondition for communication in ad hoc networks, and the method can be divided into random ones and deterministic ones according to whether the signal is sent in a probabilistic manner. Neighbor discovery methods also can be divided into synchronous methods and asynchronous methods according to whether the prior information for synchronizing joins.

In scenarios without external time synchronization and angle synchronization, algorithms based on ALOHA are usually used; however, the existing papers usually assume that each node has the same transmission probability in different directions, and only the optimal transmission probability is found. In the process of neighbor discovery of asynchronous directional ad hoc networks, self-synchronization is usually performed firstly and then neighbor discovery under synchronous conditions secondly. The paper [4] shows that the 
asynchronous neighbor discovery time is about twice the synchronous neighbor discovery time, so how to minimize the maximum average self-synchronization time is the key point to reduce the time for new nodes of joining in the directional ad hoc network and network establishment time. In this paper, we design a cooperative antenna selection method for directional antenna ad hoc networks based on ALOHA, which minimizes the maximum average time for selfsynchronization.

In this paper, we will show that with the different topologies composed of synchronized nodes, the unsynchronized nodes have different probabilities of receiving data packets from synchronized nodes in the network at different directions and locations. Based on the parameter sharing and the relative position relationship within the synchronized nodes, a cooperative antenna selection method for directional antenna ad hoc networks based on ALOHA is designed to calculate the transmission probability of nodes in different directions to minimize the maximum average time for the self-synchronization. The main contributions of this article include the following:

(1) We proved that with the different topologies composed of different nodes, the probability of receiving packets of new nodes in different directions and positions is different

(2) We designed the data exchange protocol of the nodes by transforming the problem of minimizing the maximal average self-synchronization time into the problem of minimizing the infinite norm. By this, the suboptimal transmitting probabilities at different directions are obtained, and the maximum average self-synchronization time is minimized

The rest of this paper is arranged as follows. Section 2 summarizes the related works in self-synchronization and neighbor discovery. Our assumptions and network model are described in Section 3. We firstly give the expressions of self-synchronization time of new nodes and then discuss the relationship between the self-synchronization time and their locations in Section 4. Section 5 introduces our proposed cooperative antenna selection method for directional antenna ad hoc networks based on ALOHA. Section 6 gives the experiments, parameter setup, and experimental result analysis. Finally, we summarize this work in Section 7.

\section{Related Works}

Neighbor discovery algorithms can be divided into random methods and deterministic methods according to whether the signal is sent in a probabilistic manner and also can be divided into synchronous methods and asynchronous methods according to whether the prior information for synchronizing joins. The deterministic neighbor discovery method is usually a synchronization method in which each node transmits the signal in a predetermined deterministic mechanism. The deterministic neighbor discovery method can obtain all neighbors in a fixed time.

Deterministic neighbor discovery algorithms are mainly divided into arbitration-based algorithms [5] and algorithms based on prime numbers [6, 7]. Arbitration-based algorithms divide time into a two-dimensional matrix. Each node randomly selects one row and one column as active time slots. Neighbor discovery occurs when at least two active time slots overlap with each other in two-node pairs. The algorithm based on prime numbers is mainly designed according to the Chinese remainder theorem [8], in which Disco [6], Uconnect [7], and Searchlight [9] are typical. In Disco [6], each node chooses a pair of prime numbers so that the sum of their reciprocals is set as close as possible to the required duty cycle. Then, the node will wake up with a multiple of a single prime number. If one node chooses the prime number $\left(p_{1}, p_{2}\right)$ and another node chooses the prime number $\left(p_{3}, p_{4}\right)$, the worst discovery delay between these two nodes will be $\min p_{1} p_{3}, p_{2}$ $p_{4}, p_{1} p_{4}, p_{2} p_{4}$, on the condition that the two prime numbers in a pair are not equal.

In the distributed scene, deterministic methods, which usually require prior knowledge of node synchronization and the number of neighbors (such as SMAC [10] and BMAC [11]), usually run at the cost of increasing the running time. These algorithms mainly use GPS or self-synchronization methods to achieve synchronization between two nodes. The working mechanism between nodes must be the same as each other to realize the mutual discovery. However, these methods require sending the additional data and extra power consumption to maintain synchronization within the in-network nodes. Therefore, this algorithm is not suitable for powerconstrained scenarios. In an asynchronous system, there is no global clock, and the node may be initiated at different times, so they may miss each other's transmissions. Asynchronous methods usually use random neighbor discovery methods, and each node transmits the data at a randomly selected time and finds all its neighbors at a high probability in a given time.

As for the omnidirectional antenna asynchronous neighbor discovery, McGlynn and Borbash [12] separated the neighbor discovery algorithm from the MAC protocol for the first time and defined it as an independent protocol. They proposed an asynchronous neighbor discovery protocol called birthday protocol $[12,13]$. In the paper [13], Borbash et al. studied the optimal TDMA time slot length under asynchronous conditions, under the condition that the TDMA time slot length is $T=W T_{m}$, where $T_{m}$ represents the time of sending data. For synchronous systems, $W=1$. For asynchronous systems, the paper proved that the optimal value of $W$ is 2 , and the optimal value of $P_{t}$ is the reciprocal of the number of neighbors. Vasudevan et al. [4] mapped random neighbor discovery to the coupon collector's problem. With the absence of the number of neighbor nodes and external synchronization, by analyzing the efficiency of the random discovery strategy, Vasudevan et al. assumed the following. (1) Each node has a unique ID. (2) Each node is equipped with a set of transceivers that can send or receive, but not at the same time. (3) When multiple nodes send, conflict occurs at the receiving 
node, and all packet loss is caused by the conflict. (4) The communication is considered to be bilateral. The method based on ALOHA is as follows: each node independently selects the transceiver state in each time slot, transmits at the probability of $p_{x}$, and receives at the probability of $1-p_{x}$. The papers [14, 15] proved that the optimal sending probability is $1 / n$, where $n$ is the number of nodes in the largest clique that contains this node. In the largest clique formed by $n$ nodes, the probability of node $i$ successfully transmitting data is

$$
p=p_{x}\left(1-p_{x}\right)^{n-1}=\frac{1}{n}\left(1-\frac{1}{n}\right)^{n-1} \approx \frac{1}{n e} .
$$

We use the random variable $W$ to represent the time taken for a node to discover all its neighbor nodes. The neighbor discovery process can be regarded as a sequence. Each sequence contains 1 or more time slots. $W_{m}$ represents the length of the sequence $m(0 \leq m \leq n-1)$, which represents the discovering time between the $m$-th node and the $m+1$ -th node. In the $m$-th sequence, there are still $n-m$ nodes undiscovered, so $W_{m}$ follows the geometric distribution of the parameter $(n-m) p$. Since $W=W_{0}+W_{1}+\cdots+W_{n-1}$, we have [4]

$$
E[W]=\sum_{m=0} n-1 E\left[W_{m}\right]=\sum_{m=0}^{n-1} \frac{1}{(n-m) p}=\frac{1}{p} \sum_{m=1}^{n} \frac{1}{m} \approx n e H_{n}
$$

where $H_{n}$ is the $n$-term harmonic series, $H_{n}=\sum_{m=1}^{n} 1 / m=\ln$ $n+\gamma+\varepsilon_{n}$, where $\gamma$ is the Euler-Mascheroni constant and $\varepsilon_{n}$ $\approx 1 / 2 n$; therefore,

$$
E[W]=n e\left(\ln n+\gamma+\varepsilon_{n}\right) \approx n e \ln n+\gamma n e+\frac{e}{2} .
$$

The paper [4] proved that the random variable $W$ is sharply concentrated near its mean value, so the time of neighbor discovery $W$ takes the value $\Theta(n \ln n)$ at a high probability, and it proposed a distributed neighbor discovery algorithm, in which the upper limit of neighbor discovery time is $4 n e \ln n$, under the condition of synchronization and unknowing the number of neighbors and knowing the asynchronous neighbor nodes; in the worst case, when the number of asynchronous neighbor nodes is unknown, the neighbor discovery time is $8 n e \ln n$, which is twice that under the synchronous condition.

As for communication systems such as CDMA or MIMO, which supports sending and receiving multiple packets at the same time, the paper [16] studied the neighbor discovery problem under this case. For a clique of $n$ nodes, if the number of each node sending and receiving simultaneously is up to $k$, the neighbor discovery time can be expressed as

$$
W=\Theta\left(\frac{n \ln n}{k}\right) \text { w.h.p. }
$$

Because the omnidirectional broadcast can be easily achieved, neighbor discovery using omnidirectional antennas is relatively simple. However, the difficulty of using directional antennas to realize neighbor discovery lies in the following points. (1) The beamwidth of directional antennas is limited, covering only a part of the azimuth angle. It is required to repeat the neighbor discovery scheme in different directions to cover the entire $360^{\circ}$. (2) Neighboring nodes must know what, when, and where to point their directional beams to discover each other. (3) Because of the existence of directional antenna sidelobes, two adjacent nodes may find multiple links through different directional antennas.

The neighbor discovery algorithm for wireless ad hoc networks based on directional antennas can be divided into directional transmission omnidirectional reception algorithms, omnidirectional transmission directional reception algorithms, and directional transmission directional reception algorithms according to whether directional antennas are used for transmission and reception.

The representative algorithms for directional transmission omnidirectional reception are One-Way Random (1-R) [15] and Wheeled-Iteration Neighbor Discovery. The One-Way Random algorithm is described as follows: each node in each time slot transmits at a probability of $p_{t}$ and receives at a probability of $1-p_{t}$. When the node transmits, a transmission beam is randomly selected, and the node uses omnidirectional reception. The performance analysis of the One-Way Random algorithm is described as follows: for a clique with $k$ nodes, the probability of node $i$ finding node $j$ in a time slot is [15]

$$
p_{i, j}=\frac{\theta}{2 \pi} p_{t}\left(1-\frac{\theta}{2 \pi} p_{t}\right)^{k-2}\left(1-p_{t}\right)
$$

where $p_{t}$ is the transmission probability and $\theta$ is the beamwidth. Within $t$ time slots, the probability of node $i$ finding node $j$ is [15]

$$
P_{i, j}=1-\left(1-p_{i, j}\right)^{t}
$$

According to the above two expressions, the optimal sending probability is [15]

$$
p_{t}=\frac{(2+(k-1)(\theta / 2 \pi))-\sqrt{(2+(k-1)(\theta / 2 \pi))^{2}-4 k(\theta / 2 \pi)}}{k \theta / \pi} .
$$

When the value of $k$ is large, the above formula can be simplified as $p_{t} \approx 2 \pi / k \theta$, and the neighbor discovery time is expressed as [15]

$$
W=\frac{3(\Delta+1) e \ln n}{2 \pi / \theta(1-(2 \pi /(\Delta+1) \theta))} \text { w.h.p., }
$$

where $\Delta$ is the maximum degree of the node. For omnidirectional transmission directional reception, the optimal 
transmission probability is $p_{t} \approx 2 \pi / k \alpha$, where $\alpha$ is the width of the receiving beam. As for the case where both the transmission and reception are directional, the optimal transmission probability is $p_{t} \approx 4 \pi^{2} / k \theta \alpha$, where $\alpha$ is the width of the receiving beam and $\theta$ is the width of the sending beam.

For the directional transmission omnidirectional reception algorithm, An and Hekmat [17] proposed a directional transmission omnidirectional reception neighbor discovery protocol, which adjusts the frequency of neighbor discovery attempts and the direction of neighbor discovery attempts based on the dynamics of the network. [17]. Similar to the paper [17], Ji et al. [18] proposed an integrated radar communication neighbor discovery algorithm, which makes full use of the neighbor's location information provided by radar detection, and the proposed scheme can avoid repetitive neighbor discovery attempts in the direction of potential neighbors. Both the directional transmission omnidirectional reception algorithm and the omnidirectional transmission directional reception algorithm have the following problems. (1) If an ordinary directional antenna is used, the gain of the omnidirectional antenna is much lower than that of the directional antenna, and the communication range is shorter than that of the directional transceiver mode under the same signal-to-interferenceplus-noise ratio (SINR) threshold. (2) For digital phased array antennas, although the gain of the omnidirectional receiving antenna can be the same as that of the directional receiving antenna, the cost of the digital phased array antenna is too high, and it is not suitable for scenarios where the size, weight, and power consumption are limited. Therefore, this paper focuses on how to design a scanning transmission mechanism based on directional antennas without external time synchronization and angle synchronization. This mechanism only uses narrow beam transmission and reception and reduces the maximum average self-synchronization time as much as possible.

For the neighbor discovery algorithms for directional transmitting and directional receiving, they can also be divided into synchronization-based methods [19-21] and random-based methods $[15,22]$, according to different strategies. The synchronization-based method assumes that the time of the node has been synchronized and the directional information is included. The synchronization-based method [19-21] introduces a discovery strategy in which all nodes are synchronized in the sending and receiving directions selected, but the decision of sending or listening is at will. Park et al. [23] proposed a mathematical model to analyze the neighbor discovery strategy in ad hoc networks in which only directional pattern antennas are used and proved that the fully synchronized method under the condition that the sector size equals the antenna beamwidth performed better than the random strategy [23]. Zhang [24] proposed a synchronizationbased scanning algorithm based on external time synchronization and angle synchronization to realize neighbor discovery. Xiong et al. [21] considered the case of the existence of multiple nodes in a directional beam and proposed a scan-based algorithm (SBA) for neighbor discovery. The key idea of SBA is scanning a series of directions, and each scanning should cover each antenna direction and must not be repeated. At the beginning of each scanning, the node randomly enters the transmission mode at some probability and enters the lis- tening mode at some probability. The node should keep its mode unchanged until finishing a scanning; when sending, the node sends in each specified direction. The broadcast packet is a predefined sequence in potential neighbor detection directions. When listening, the node also waits for broadcast packets in the specified direction. If a broadcast packet is received, it will reply with a confirmation packet.

The synchronization-based neighbor discovery algorithm requires time synchronization information and angle synchronization, which is not difficult to obtain in practical applications. In this situation, random methods are usually used. Based on the random method, assuming that there is no time synchronization and pointing information between nodes, probabilistic scheduling of sending and receiving in randomly selected directions is developed. The paper [22] provided a detailed analysis of neighbor discovery protocols with 2-way communication when using directional antennas. Based on this analysis, a design of a random 2-way neighbor discovery algorithm using selective feedback is proposed. The results show that the node needs $\Theta\left(n^{2} / k\right)$ to discover $n$ neighbors at $k$ antenna sectors, which is a significant performance improvement over pure randomized algorithms. They also extend this scheme to practical application, where the number of neighbors is unknown, and show that the performance reduces by no more than $4 / 3$.

Time synchronization algorithms can be divided into direct methods and methods with messages exchanged. In direct methods, an unsynchronized node updates its time directly when packets for synchronized ones are received. In methods with messages exchanged, the transmission delay is calculated by the handshakes between nodes, in which Round-Trip Synchronization [25], Network Time Protocol (NTP) [26], Reference Broadcast Synchronization (RBS) [25], Lightweight Time Synchronization (LTS) [27], Timingsync Protocol for Sensor Networks (TPSN) [28], and Flooding Time Synchronization Protocol (FTSP) [29] are typical. No matter which time synchronization algorithm is adopted, the first step of self-synchronization for new nodes is to receive a packet for synchronized ones. Without external time synchronization and angle synchronization, the unsynchronized node usually takes a long time to receive the first packet from synchronized ones in directional ad hoc networks. Thus, the key point of minimizing the synchronization time in directional ad hoc networks is to reduce the waiting time for an unsynchronized node to receive the first data packet of synchronized ones.

The existing random-based neighbor discovery algorithms for directional ad hoc networks without external time synchronization and angle synchronization can be improved in two ways. (1) The existing neighbor discovery algorithms only consider that each node makes decisions independently and do not consider the interaction of cooperation information within the nodes. (2) Whether One-Way Random or polling iterative algorithm, the node scans the beam pointing at $360^{\circ}$ at equal probability. In the condition of no topological prior information, this approach is optimal, but once topological prior information is present, the equal probability scanning may not be optimal. Based on the above two points, we 
propose a cooperative antenna selection method for directional antenna ad hoc networks based on ALOHA, without external time synchronization and angle synchronization.

\section{System Model}

Before presenting the proposed cooperative antenna selection method for directional antenna ad hoc networks based on ALOHA, we briefly describe our assumptions and network model.

First of all, we have the following assumptions:

(1) Each node can generate $K$ beams to achieve $360^{\circ}$ coverage, and the width of each beam is the same as $\alpha=2 \pi / K$

(2) Each node can only select one of the antenna beams for transmission or reception at the same time

(3) TDMA is adopted

(4) The nodes have no external time synchronization

(5) The communication range of each node is $R$

(6) The nodes have no angle synchronization, and the antenna placement direction of each node is random, and each array is numbered clockwise

(7) We adopt such directional antenna interference model that node $A$ and node $B$ send data to node $C$ at the same time; as shown in Figure 1, the conditions for node $A$ and node $B$ not to interfere with each other are

$$
\angle A C B>(1+\Delta) \alpha,
$$

where $\Delta$ is the interference parameter, which is used to measure how far apart the two beams will not interfere with each other, which depends on the size of the sidelobes of the receiving and sending beams, SINR threshold, and antiinterference ability of the physical layer.

The interference model is shown in Figure 1. It can be seen that the physical meaning of $\Delta \bullet \alpha$ is the minimal angle shifting away from the edge of the receiving beam (assumed that node $C$ point to $A$ ) that the signals will not interfere with each other. Then, we perform network modeling.

We consider the similar network model in the paper [30] on the Euclidean two-dimensional plane, using $\Phi=\left\{X_{i}, e_{i}\right.$, $\left.\omega_{i}, \varphi_{i}, F_{i}, T_{i}\right\}$ to represent the problem in $\mathbb{R}^{2}$. The density on the plane is the homogeneous Poisson point process of $\lambda$, where

(1) $\Phi=\left\{X_{i}\right\}$ denotes the polar coordinates of the nodes

(2) $\left\{e_{i}\right\}$ is the MAC indicator of node $i$, and $e_{i}=1$ if node $i$ is allowed to transmit and 0 otherwise

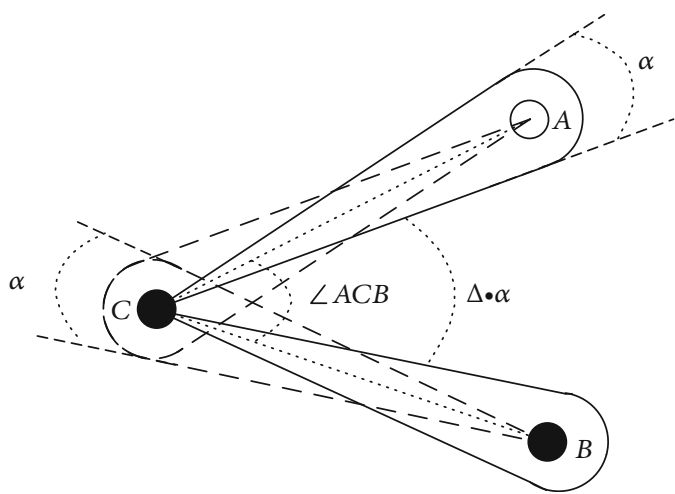

FIGURE 1: Directional antenna interference model

(3) $\omega_{i}\left(0 \leq \omega_{i}<2 \pi\right)$ denotes the angle of the first beam pointing relative to north, and $\omega_{i}$ obeys uniform distribution $[0,2 \pi)$

(4) $\varphi_{i}\left(0 \leq \varphi_{i}<2 \pi\right)$ denotes the angle of node $i$ beam pointing relative to north

(5) $\left\{F_{i}=\left(F_{i}^{j}\right)\right\}$ denotes the virtual power emitted by node $i$, which means the product of the effective power of transmitter $i$ and of the random fading from this node to the receiver. A special important case consists in assuming constant emitted power and Rayleigh fading which implies exponential $F$

(6) $T_{i}$ denotes the SINR threshold of node $i$, and we assume that the SINR thresholds are the same, $\forall i$ $T_{i}=T$

Omnidirectional path loss represents $l(\bullet)$ :

$$
l(u)=(A u)^{-\beta}, \quad A>0, \beta>2 .
$$

The condition for node $y$ to receive node $x$ is

$\operatorname{SINR}_{x y}\left(\theta, \varphi_{x}, \varphi_{y}\right)=\frac{F_{x}^{y} \cdot l(|x-y|) \cdot A F_{x}\left(\varphi_{x}\right) \cdot A F_{y}\left(\varphi_{y}\right)}{W+I_{\Phi^{1}}\left(\theta, \varphi_{y}\right)} \geq T$.

$W$ represents additive Gaussian white noise, and $I_{\Phi^{1}}$ $\left(\theta, \varphi_{y}\right)$ denotes the interference signal received when the node $y$ beam points to $\varphi_{y} \cdot A F_{i}\left(\varphi_{i}\right)$ denotes the antenna gain of node $i$ in the direction of $\varphi_{i}$.

$$
I_{\Phi^{1}}\left(\theta, \varphi_{y}\right)=\sum_{j \in \Phi \wedge^{1}} F_{j}^{y} \cdot l(|j-y|) \cdot A F_{j}\left(\varphi_{j}\right) \cdot A F_{y}\left(\varphi_{y}\right)
$$

$\Phi \wedge^{1}$ represents the set of nodes that are in the transmitting state at the current moment. It can be seen from formula (11) that the signal-to-noise ratio at the receiving nodes depends on the interference from other nodes 
and the product of the gains of the transmit and receive antennas. The product of the gains of the transmit and receive antennas at the same time depends on the angle of the transmit and receive beams, namely,

$$
A F_{x}\left(\varphi_{x}\right) \cdot A F_{y}\left(\varphi_{y}\right)=f\left(\varphi_{x}-\varphi_{y}\right)
$$

The same as formula (9), assume that the node uses a uniform linear array (ULA) with 20 elements, and the "Hanning" window is used. The normalized antenna gains at $0^{\circ}$ are shown in Figure 2.

Taking the antenna gains shown in Figure 2 as an example, it can be seen that the $3 \mathrm{~dB}$ beamwidth is about $9^{\circ}$, and the gain of the antenna offset $20^{\circ}$ away from the main beam is about $-40 \mathrm{~dB}$. If the physical layer works normally under the interference-to-signal ratio (ISR) of $-40 \mathrm{~dB}$, the interference parameter is about 3.4. It can be seen that the directional antenna interference model in this paper (9) is in line with the actual situation. The interference parameter depends on the gain of the antenna beam sidelobe, the SINR threshold, and the anti-interference ability of the physical layer.

\section{The Maximal Average Synchronization Time for Directional Ad Hoc Networks}

Without external time synchronization and angle synchronization, the unsynchronized node usually takes a long time to synchronize with the existing nodes due to the narrow beams in directional ad hoc networks. In this section, firstly, we will give the expressions of synchronization time; secondly, we will show that with the different topologies composed of synchronized nodes, the unsynchronized nodes have different probabilities of receiving data packets from synchronized nodes in the network at different directions and locations.

For the asynchronous directional TDMA ad hoc network without external time synchronization and angle synchronization, if a phased array antenna is used, since the distance measurement can be performed through the phased array antenna, the receiving node only needs to receive one packet from synchronized nodes; thus, the node can achieve time synchronization. At this time, the self-synchronization time is the waiting time for the unsynchronized node to receive the first data packet of synchronized nodes. If a common directional antenna is used, in which distance measurement cannot be achieved through a single information transmission, the usual method is to use a three-way handshake protocol similar to NTP to achieve network time synchronization, the first handshake to achieve coarse synchronization and the second and third handshakes to achieve distance measurement, achieve fine synchronization, and finally achieve complete network time synchronization; the latter is usually a fixed value (related to the specific protocol design). Based on the above two points, the self-synchronization time in a directional antenna ad hoc network can be expressed as

$$
T(\tau)=\tau+\xi
$$

In formula (14), $\tau$ represents the waiting time for the unsynchronized node to receive the first data packet of synchronized ones, that is, coarse synchronization time, and $\xi$ represents the fine synchronization time. When the node has the ranging function through one signal transmission, for example, a phased array antenna is used. At this time, the fine synchronization time $\xi$ is 0 ; otherwise, $\xi$ is a fixed value related to the protocol design and has nothing to do with $\tau$. Therefore, minimizing the maximum average self-synchronization time of the unsynchronized node is a problem, which is equivalent to minimizing the maximum $\tau$.

In the following, we will prove that in a directional antenna ad hoc network, for a network where multiple nodes have completed neighbor discovery, depending on the topology, the probability of receiving a data packet from synchronized nodes is different according to geometric position relative to the synchronized nodes. We first consider the simplest case, when there is only one node that has been ynchronized; at this time, because the unsynchronized nodes are subject to the homogeneous Poisson point process with a density of $\lambda$, for the nodes that have been synchronized, at each direction of $360^{\circ}$, the probability of the number of unconnected nodes is the same. At this time, the optimal transmission strategy is equal probability transmission in each direction.

When there are two connected nodes, the distance between the connected nodes $A$ and $B$ is $d<R$. The position of node $A$ is the origin of the polar coordinate. The direction from node $A$ to node $B$ can be the polar coordinate direction to establish a polar coordinate system. The coordinate of node $A$ is $(0,0)$, the coordinate of node $B$ is $(d, 0)$, and we use $\operatorname{ta}(\theta)$ and $t b(\theta)$ to represent the transmission probability of node $A$ and node $B$ in the direction of $\theta(0 \leq \theta<2 \pi)$. Since the unsynchronized node does not know the geographic location and geometric distribution of the synchronized nodes, the receiving probability of the unsynchronized node in the $360^{\circ}$ direction is the same, that is, $\forall \theta \in[0,2 \pi) r c(\theta)=\alpha / 2 \pi$ $=1 / K$. At this time, the probability that the unsynchronized node $C(\rho, \theta)$ will receive the data packets of node $A$ and node $B$ is shown in the following expression:

$$
p(\rho, \theta)=\left\{\begin{array}{l}
\frac{t a\left(\theta_{A C}\right)}{K}, \quad C \in \Omega_{1}, \\
\frac{\left(t a\left(\theta_{A C}\right)\left(1-t b\left(\theta_{B C}\right)\right)+t b\left(\theta_{B C}\right)\left(1-t a\left(\theta_{A C}\right)\right)\right.}{K}, \quad C \in \Omega_{2}, \\
\frac{\left(t a\left(\theta_{A C}\right)+t b\left(\theta_{B C}\right)\right)}{K}, \quad C \in \Omega_{3,} \\
\frac{t b\left(\theta_{B C}\right)}{K}, \quad C \in \Omega_{4}, \\
0, \quad C \in \Omega_{5} .
\end{array}\right.
$$

Among them, the real plane $\Omega$ is divided into five regions according to the different geometric positions of the unsynchronized node $C$ and the synchronized nodes $A$ and $B$, as shown in expression (16), where $R=300, \Delta=2$, and $K=30$. The regional distribution when the distance between two synchronized nodes is set to be 100 and 200 is shown in Figure 3. 


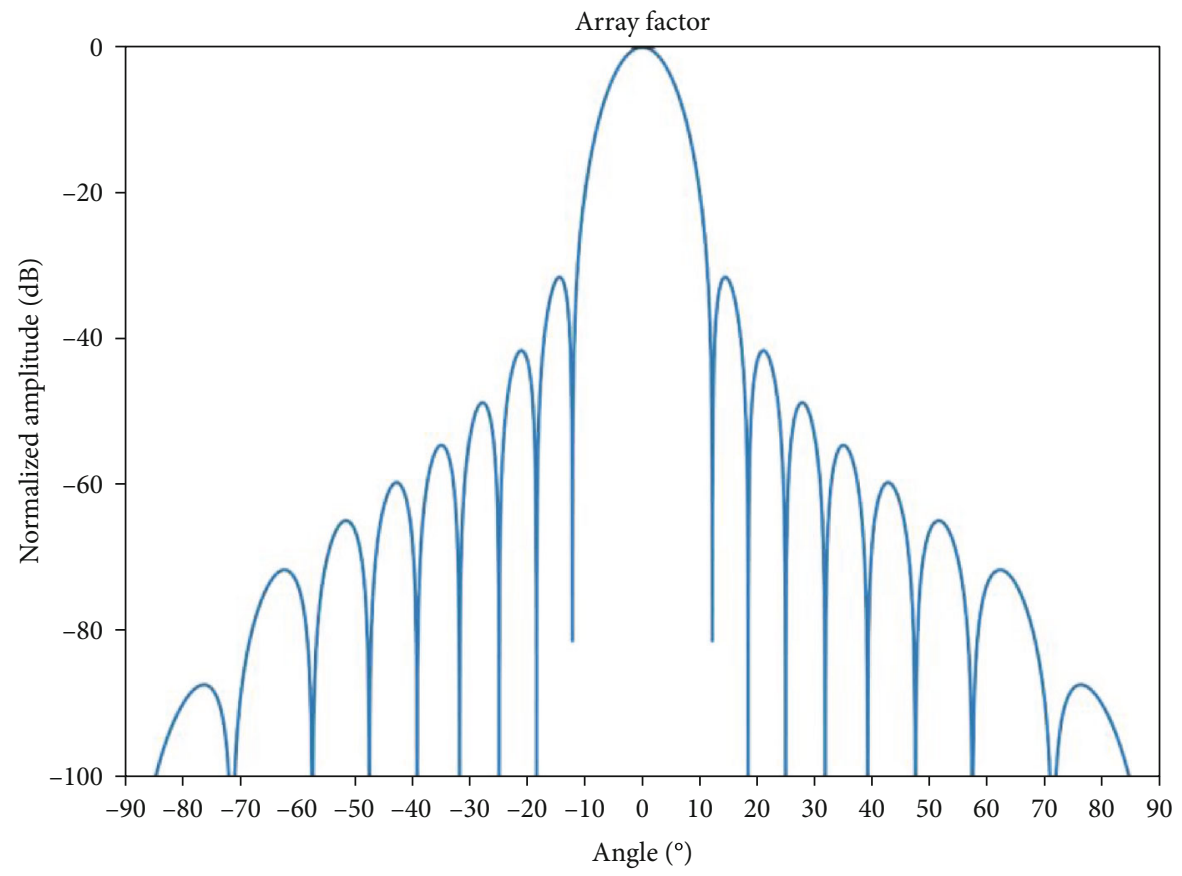

FIgURE 2: The normalized antenna gains of a uniform linear array (ULA) with 20 elements by the "Hanning" window.

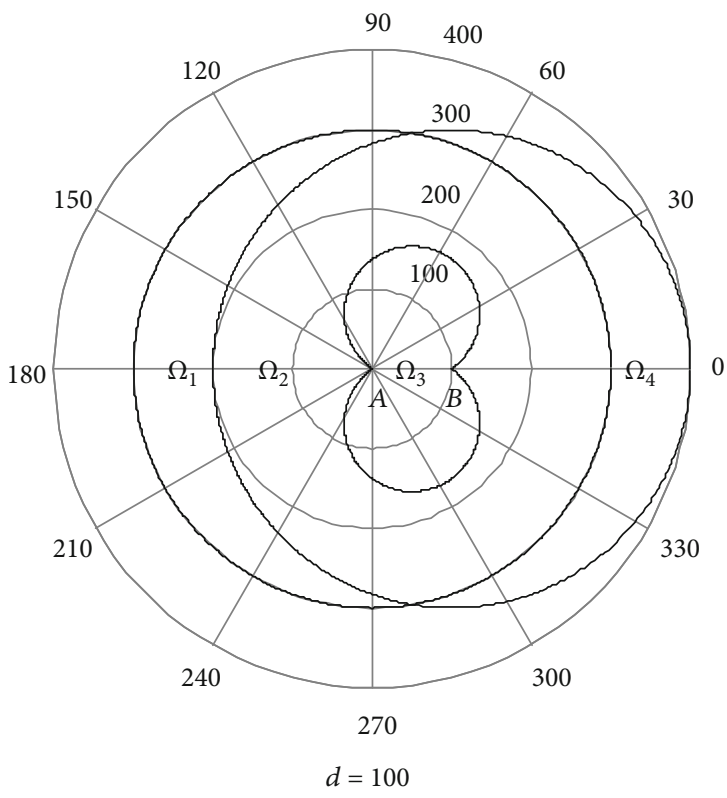

(a)

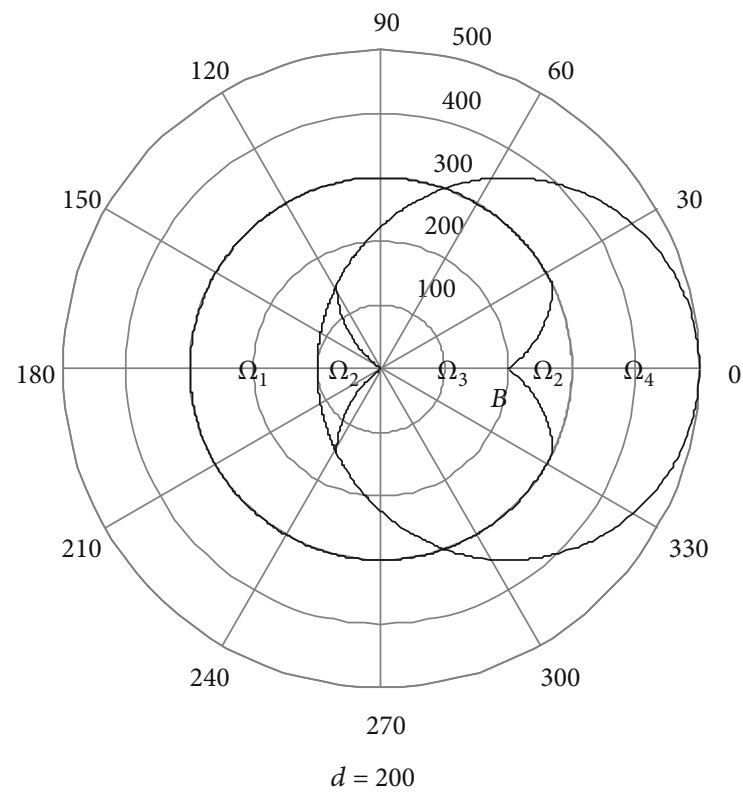

(b)

FIGURE 3: The probability distribution regions of the unsynchronized node successfully receiving the data packet of the two synchronized nodes. (a) The case when the distance between two synchronized nodes is 100. (b) The case when the distance between two synchronized nodes is 200 . 


$$
\begin{gathered}
\Omega=\Omega_{1} \cup \Omega_{2} \cup \Omega_{3} \cup \Omega_{4} \cup \Omega_{5}, \\
\Omega_{1}=\left\{(\rho, \theta) \mid \rho \leq R, \sqrt{\rho^{2}+d^{2}-2 \rho d \cos (\theta)}>R\right\}, \\
\Omega_{2}=\left\{(\rho, \theta) \mid \rho \leq R, \sqrt{\rho^{2}+d^{2}-2 \rho d \cos (\theta)} \leq R, \arccos \left(\frac{\rho-d \cos (\theta)}{\sqrt{\rho^{2}+d^{2}-2 \rho d \cos (\theta)}}\right)<\alpha(1+\Delta)\right\}, \\
\Omega_{3}=\left\{(\rho, \theta) \mid \rho \leq R, \sqrt{\rho^{2}+d^{2}-2 \rho d \cos (\theta)} \leq R, \arccos \left(\frac{\rho-d \cos (\theta)}{\sqrt{\rho^{2}+d^{2}-2 \rho d \cos (\theta)}}\right) \geq \alpha(1+\Delta)\right\}, \\
\Omega_{4}=\left\{(\rho, \theta) \mid \rho>R, \sqrt{\rho^{2}+d^{2}-2 \rho d \cos (\theta)} \leq R\right\}, \Omega_{5}=\Omega-\left(\Omega_{1} \cup \Omega_{2} \cup \Omega \cup \Omega_{4}\right) .
\end{gathered}
$$

Nodes in regions $\Omega_{1}$ are in the communication range of one node and cannot communicate with the other. If the unsynchronized node is in the receive mode, the nearest synchronized node is in the transmit mode, and the unsynchronized node and the nearest synchronized node point to each other, and the unsynchronized node can receive a data packet of synchronized ones. So, the probability that the unsynchronized nodes in regions $\Omega_{1}$ will receive data packets of synchronized ones is $\operatorname{ta}\left(\theta_{A C}\right) / K$. $\theta_{A C}$ denotes the direction that node $A$ points to node $C$. Similarly, the probability for the unsynchronized nodes in regions $\Omega_{4}$ is $t b\left(\theta_{B C}\right) / K$.

Nodes in regions $\Omega_{2}$ are in the communication range of both nodes, and the angle between the unsynchronized nodes and the two synchronized nodes is less than $\alpha(1+\Delta)$, which means the two synchronized nodes will interfere with each other when they are all in the transmit mode and point to the same unsynchronized nodes. If one of the synchronized nodes is in the transmit mode and points to the unsynchronized node and the other not, the unsynchronized node can receive a data packet of synchronized ones. So, the probability for the unsynchronized nodes in regions $\Omega_{2}$ is $\left(\operatorname{ta}\left(\theta_{A C}\right)\right.$ $\left.\left(1-t b\left(\theta_{B C}\right)\right)+t b\left(\theta_{B C}\right)\left(1-t a\left(\theta_{A C}\right)\right)\right) / K$.

Nodes in regions $\Omega_{3}$ are in the communication range of both nodes, and the angle between the unsynchronized nodes and the two synchronized nodes is not less than $\alpha(1+\Delta)$, which means the two synchronized will never interfere with each other. So, the probability for the unsynchronized nodes in regions $\Omega_{3}$ is $\left(\operatorname{ta}\left(\theta_{A C}\right)+t b\left(\theta_{B C}\right)\right) / K$.

From the above, we get the expressions of (15) and (16). Then, we will analyze the situation with multiple nodes.

When there are $N$ nodes in the network, the polar coordinates of the synchronized node $N_{i}$ are $N_{i}\left(\rho_{i}, \theta_{i}\right)$, and $t_{i}(\theta)$ denotes the transmit probability of node $i$ in the direction of $\theta(0 \leq \theta<2 \pi)$. We use $l_{i}$ to represent the distance between the unsynchronized node and the synchronized node $N_{i}$ and use $\phi_{i j}$ to represent the angle formed by node $i$ and node $j$ and the unsynchronized node. Then, the proba-

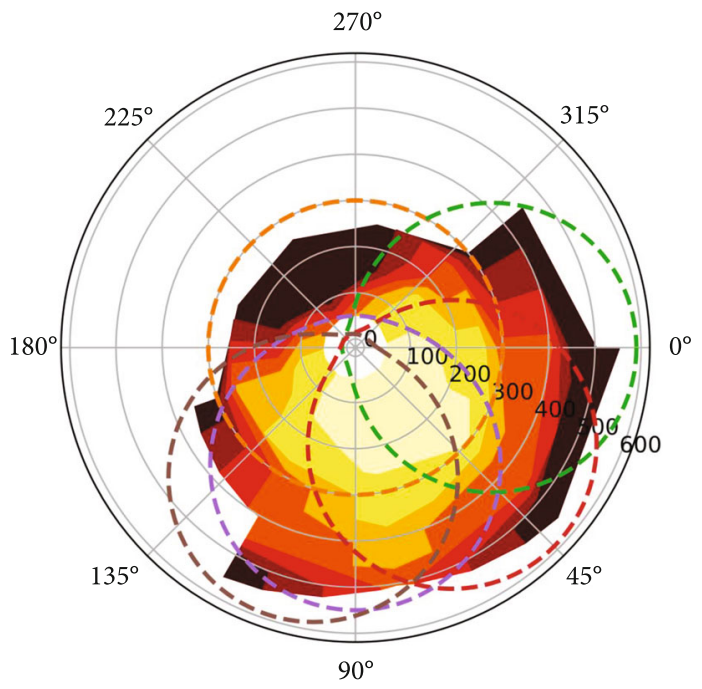

FIgURE 4: The relationship between the geometric position of the unsynchronized node relative to synchronized nodes and the distribution of the waiting time of the first packet received; the darker color represents the longer waiting time.

bility that the unsynchronized node successfully received packets from synchronized nodes is expressed as

$p(\rho, \theta)=\frac{1}{K}\left[\sum_{i=1, l_{i}<R}^{i=N}\left(t_{i}(\theta) \prod_{j=1, j \neq i, l_{j}<R, j \in \Psi_{i}}\left(1-t_{j}(\theta)\right)\right)+\sum_{j=1, j \neq i, l_{j}<R, j \in \widehat{\Psi}_{i}}^{j=N} t_{j}(\theta)\right]$.

$\Psi=\Psi_{i}+\widehat{\Psi}_{i}$ denotes the set of all connected nodes, and $\Psi_{i}$ represents the set of all nodes whose angle between the unsynchronized node and node $i$ is less than $\alpha(1+\Delta)$ given the position of the unsynchronized $(\rho, \theta)$, and $\Psi_{i}$ and $\widehat{\Psi}_{i}$ can be expressed as 


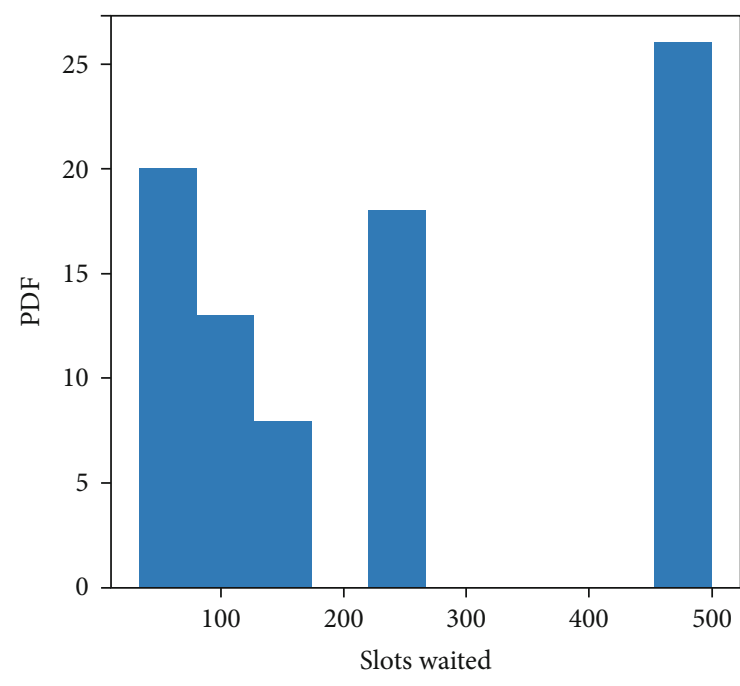

(a)

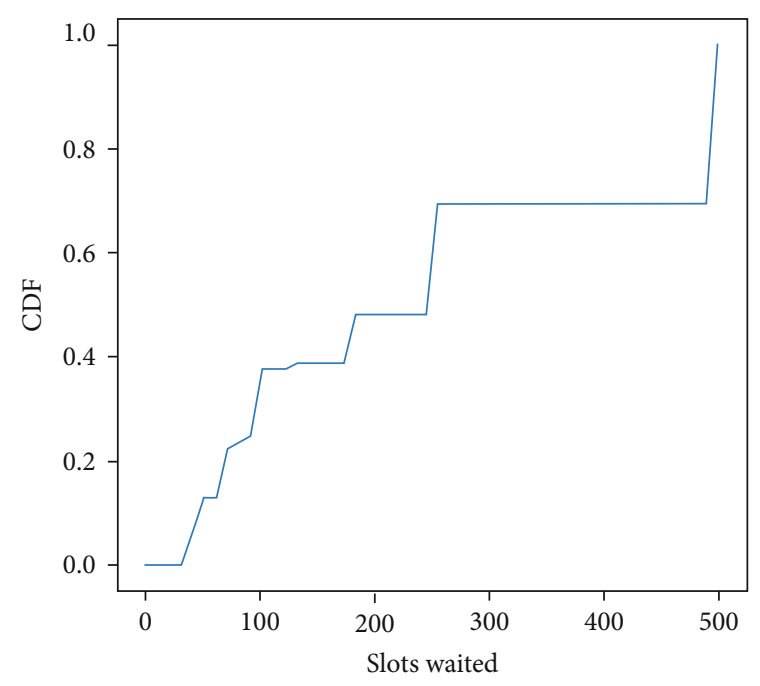

(b)

FIGURE 5: The probability density function and cumulative distribution function curves of the average waiting time of unsynchronized nodes. (a) The probability density function curve of the average waiting time of unsynchronized nodes. (b) The cumulative distribution function curve of the average waiting time of unsynchronized nodes.

$$
\begin{gathered}
\Psi_{i}=\left\{N_{j} \mid N_{i}, N_{j} \in \Psi, \phi_{i j} \geq \alpha(1+\Delta)\right\}, \\
\widehat{\Psi}_{i}=\left\{N_{j} \mid N_{i}, N_{j} \in \Psi, \phi_{i j} \geq \alpha(1+\Delta)\right\} .
\end{gathered}
$$

According to the paper [22], assuming that the probability of a node successfully receiving a data packet in one time slot is $p$, the waiting time for the node to receive the first data packet obeys the exponential distribution of parameter $p$; thus, the expectation of the waiting time for the unsynchronized node receiving the first packet is

$$
E(T)=\frac{1}{p} .
$$

We simulated the distribution of the expectation of the waiting time. In our simulation, there are five synchronized nodes, $R=300, \Delta=2$, and $K=30$, and the coordinates of nodes are $N_{1}(0,0), N_{2}(290,0), N_{3}(290, \pi / 4), N_{4}(250, \pi / 2)$, and $N_{5}(290,3 \pi / 5)$, respectively; each node uses the optimal transmit probability, and the transmit probability of $K$ directions is the same. Figure 4 shows the relationship between the geometric position of the unsynchronized node relative to synchronized nodes and the distribution of the waiting time of the first packet received; the darker color represents the longer waiting time.

Figure 5 shows the probability density function (PDF) curve and cumulative distribution function (CDF) curve of the average waiting time of the unsynchronized node under the geometric position distribution of the five nodes shown in Figure 4. It can be seen in Figure 5(a) that the probability density function (PDF) of the average waiting time of the unsynchronized node is discrete. If the traditional method with equal probability of different directions is adopted, the expectation of the waiting time for the first packet reception by unsynchronized nodes is discrete and finite, because the probability of the first packet reception by unsynchronized nodes is discrete and finite according to the regions divided by the communication range of multiple nodes as shown in expression (16) and Figure 3, and the probability of the first packet reception by unsynchronized nodes in the same region is the same, which is related to the area of each region. Because the unsynchronized nodes are subject to the homogeneous Poisson point process with a density of $\lambda$, the larger the area of the region is, the larger the PDF value of the corresponding waiting time. It can be seen that according to the difference between the geometrical positions of the unsynchronized node and the synchronized node, the waiting time gap of the first data packet reception from the synchronized node is relatively large. When the unsynchronized node is at the edge of the coverage of the networked node, the waiting time is relatively large, and when it is relatively central, the waiting time is relatively small.

\section{Proposed Cooperative Antenna Selection Method for Directional Antenna Ad Hoc Networks Based on ALOHA}

From the previous section, we know that the traditional method with equal transmit probability is not the optimal choice in ad hoc networks based on directional antennas. In this section, we will propose a cooperative antenna selection method for directional antenna ad hoc networks based on ALOHA, which minimizes the maximum synchronizing time in the network. According to expressions (17) and (20), we know that given the coordinates of synchronized and unsynchronized nodes, the expectation of synchronizing time is 


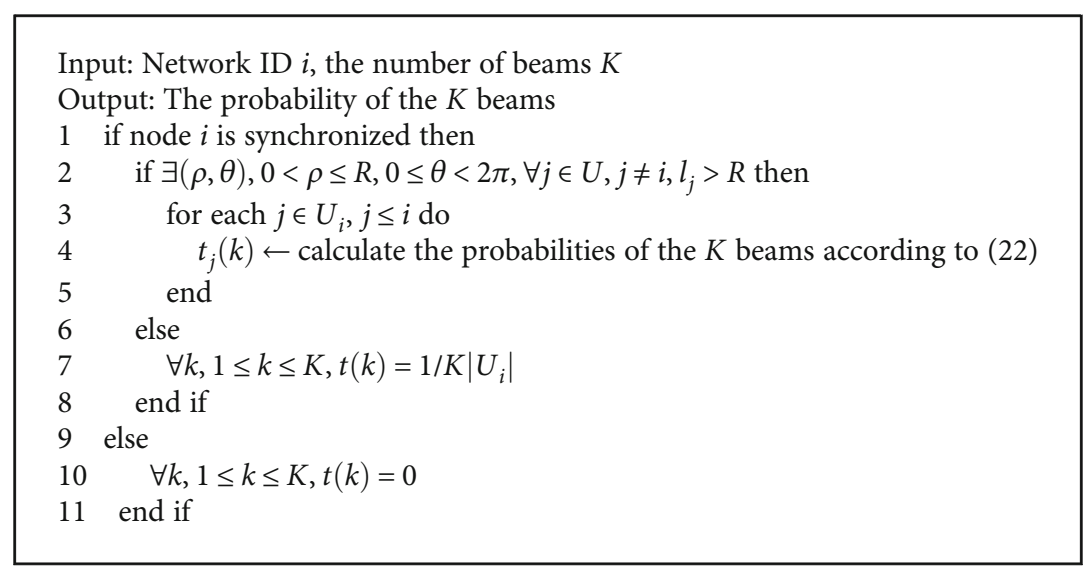

Algorithm 1: The proposed cooperative antenna selection algorithm.

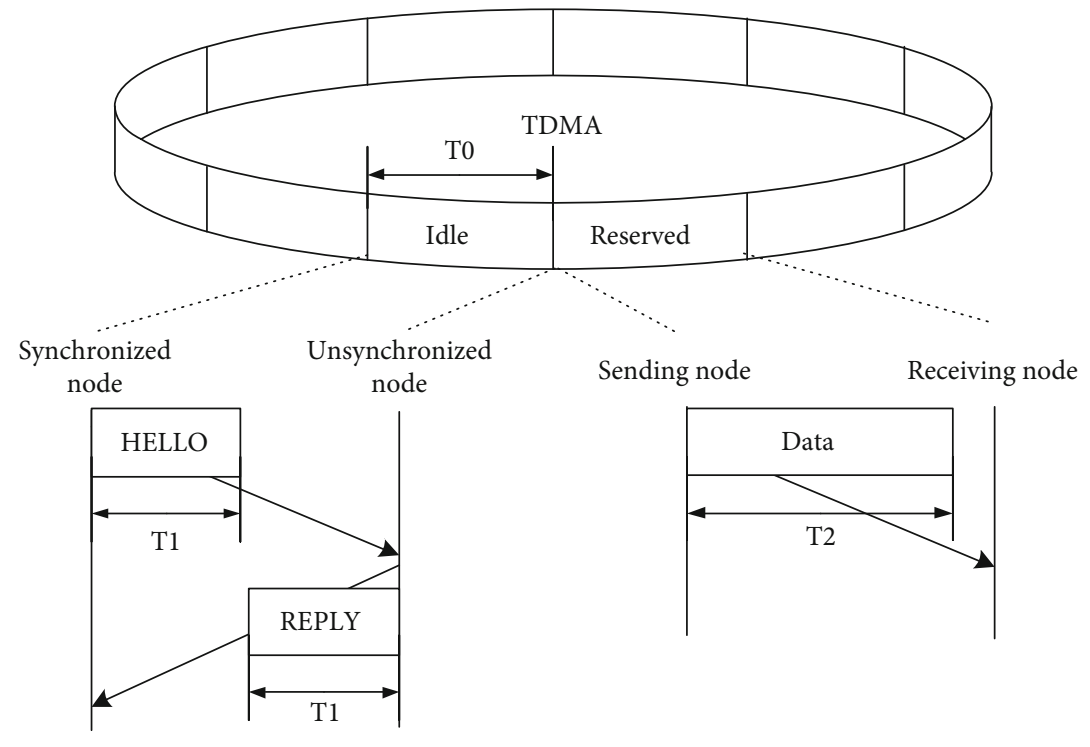

FiguRE 6: TDMA scheme.

$E(T(\rho, \theta))=K\left[\sum_{i=1, l_{i}<R}^{i=N}\left(t_{i}(\theta) \prod_{j=1, j \neq i, l_{j}<R, j \in \Psi_{i}}\left(1-t_{j}(\theta)\right)\right)+\sum_{j=1, j \neq i, l_{j}<R, j \in \Psi \wedge_{i}}^{j=N} t_{j}(\theta)\right]^{-1}$.

Therefore, the problem of minimizing the maximum synchronizing time in the network can be expressed as the problem of minimizing the infinite norm of the sequence $E(T(\rho, \theta))$, which can be expressed by the optimization problem of the following formula:

$$
\begin{gathered}
\underset{t_{i}(\theta)}{\operatorname{minimize}\|E(T(\rho, \theta))\|_{\infty}} \\
\int_{0}^{2 \pi} t_{i}(\theta) \mathrm{d} \theta \leq \frac{1}{\left|U_{i}\right|} \\
\text { subject to } \forall \theta, 0 \leq \theta<2 \pi, 0 \leq t(\theta) \leq 1 \\
0<\rho \leq R .
\end{gathered}
$$

$U_{i}$ represents the ordered set of all neighbors including node $i$ itself, and formula (22) can be solved by convex optimization methods. Note that when the unsynchronized node is in the geometric center of the synchronized node, the waiting delay is small, and when it is at the edge, the waiting delay is large. Therefore, we propose a cooperative antenna selection method for directional antenna ad hoc networks based on ALOHA, as shown in Algorithm 1, where $U$ denotes the collection of all synchronized nodes that have joined the network. For unsynchronized nodes, the receiving probability is 1 , and the sending probability is 0 ; for the synchronized nodes, if there is a point in the communication coverage of itself but not in the communication range of other synchronized nodes, then follow the node number from small to large and calculate the transmit probability of different beams of the corresponding node according to formula (22), until the transmit probabilities of different beams of the node are obtained; otherwise, the node transmits with equal probability, that is, $t(k)=1 / K\left|U_{i}\right|, 1 \leq k \leq K$.

For node $i$, formula (22) requires the polar coordinates $N_{j}\left(\rho_{j}, \theta_{j}\right)$ and the transmission probability $t_{j}(\theta)$ of each 


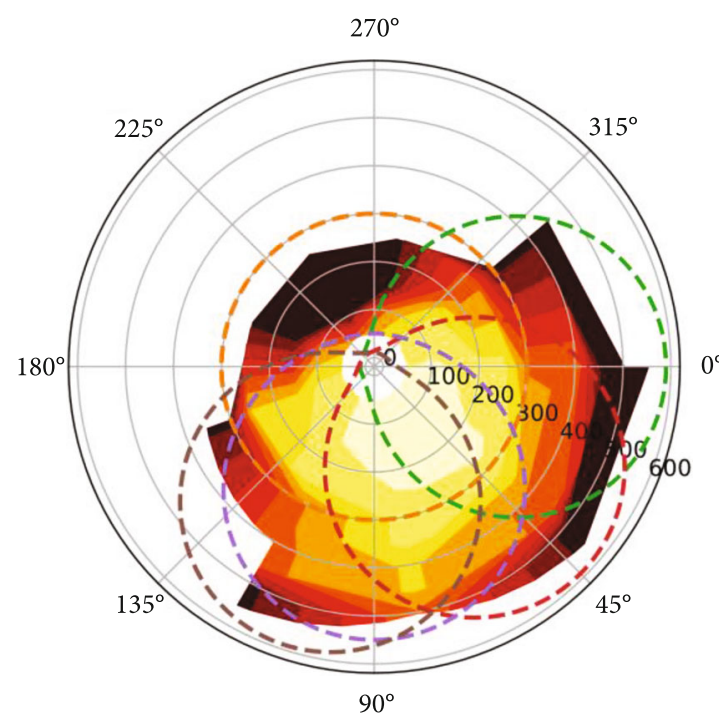

(a)

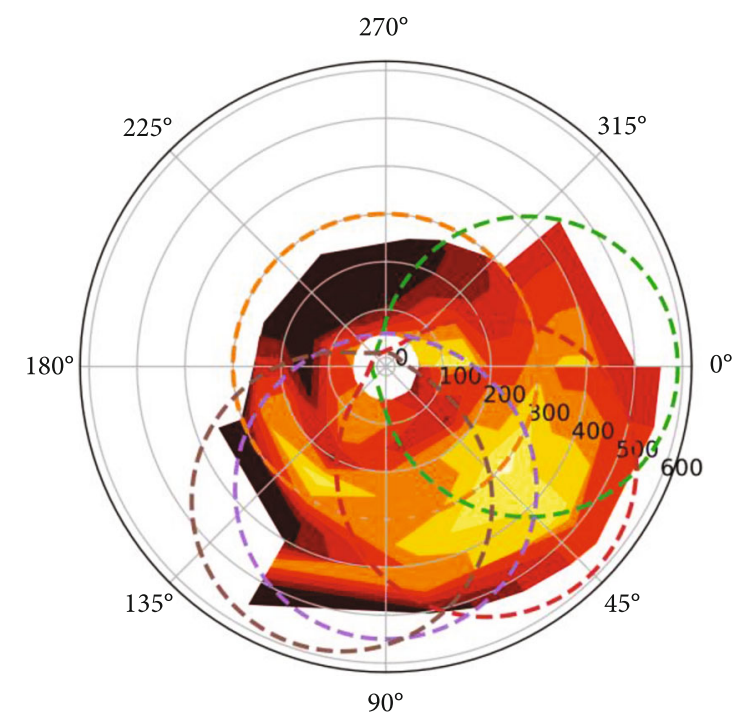

(b)

FIGURE 7: The relationship between the average waiting time to be synchronized and the geometric distribution of the synchronized node. (a) The result of the traditional method with equal transmit probability. (b) The result of the method proposed in this paper.

neighbor node $j$ in $U_{i}$. This is usually not difficult to obtain in the ad hoc network protocol. For example, we can design a TDMA scheme as shown in Figure 6, in which nodes send HELLO packets with a certain probability in idle time slots, and the selection of the transmission and reception probability can refer to the existing literature (such as [22]). If a node that has not been synchronized receives a HELLO packet, it immediately sends a REPLY packet to the receiving direction; data services can be sent or received in the reserved time slot. The time slot length T0, the HELLO packet and REPLY packet length T1, and the data packet length T2 satisfy the following relationship:

$$
\mathrm{T} 0=\mathrm{T} 2+\frac{R}{c}+\epsilon=2 \mathrm{~T} 1+2 \frac{R}{c}+2 \epsilon
$$

$R$ is the maximum communication distance, $c$ is the speed of light, and $\epsilon$ is the guard time used for receiving and transmitting switching.

Through the two handshakes of HELLO and REPLY, time synchronization and distance measurement can be carried out through time synchronization algorithms such as NTP. If a phased array antenna is equipped, the DOA of the neighbors can be estimated through the two handshakes; otherwise, the number of the directional beams of the neighbors can be obtained. Let the origin of the polar coordinate be the location of the node itself, and let the polar coordinate direction be the direction of the first beam. The polar coordinates of neighbors are obtained. The HELLO and REPLY packets include the sending probabilities of different directions arranged from a specific node. Thus, the input requirements of the algorithm proposed in this paper can be met.

\section{Experiment and Results}

The cooperative antenna selection method for directional antenna ad hoc networks based on ALOHA proposed in this paper is simulated in the situation shown in Figure 4. In our simulations, there are five synchronized nodes, $R=300, \Delta=2$ , and $K=30$, and the coordinates of nodes are $N_{1}(0,0), N_{2}$ $(290,0), \quad N_{3}(290, \pi / 4), \quad N_{4}(250, \pi / 2)$, and $N_{5}(290,3 \pi / 5)$, respectively. The problem of formula (22) is solved by the open-source GEKKO library $[31,32]$ and the IPOPT tool. Figure 7 shows the relationship between the waiting time to be synchronized and the geometric distribution of the synchronized nodes. Figure 8 is a comparison diagram of PDF and CDF of the proposed methods and the traditional method in which equal transmit probability is adopted.

The transmit probabilities at different beams of node 1 are shown in Figure 9. It can be seen from Figure 7 that using the method proposed in this paper, the coarse synchronization time of new nodes at the edge of the network is reduced. By adopting the proposed method, the transmit probabilities of different directions are different, so the average waiting time of the unsynchronized node in the same region is different. So, it can be seen from Figure 8(b) that the PDF curve of the proposed method is no longer discrete, and the average waiting time of the unsynchronized node is continuous because the probability of the first packet reception by unsynchronized nodes varies according to their geographic location. It can be seen from Figure 8 that under the traditional method using equal transmit probability, the maximum waiting delay of nodes to be synchronized is 500 time slots. After adopting the method proposed in this paper, the maximum waiting delay is 197 time slots; thus, the maximum average time for the new nodes to be synchronized is reduced by $60 \%$. It can be seen from the CDF curve that using the method proposed in this paper, the waiting time of the 


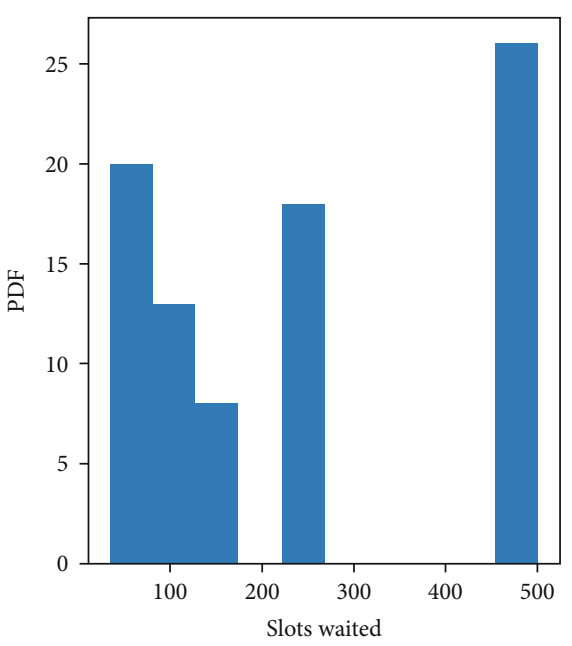

(a)

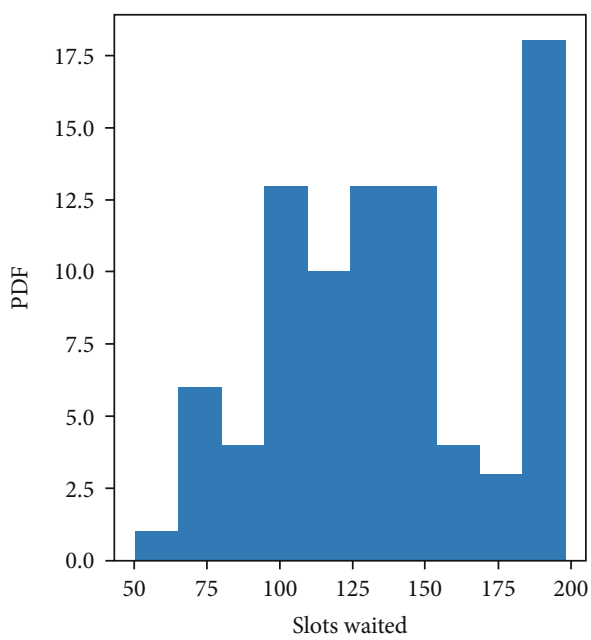

(b)

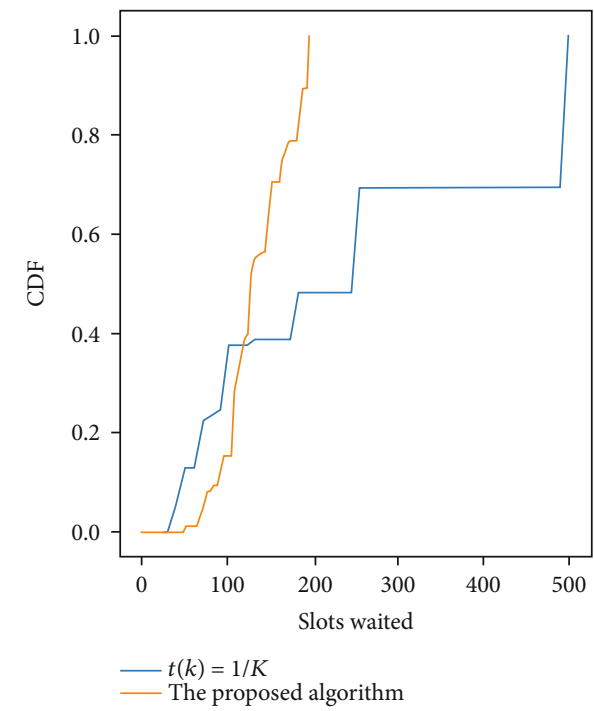

(c)

FIgURE 8: PDF and CDF comparison of the two methods. (a) The PDF of average waiting time slots of the traditional method with equal transmit probability. (b) The PDF of average waiting time slots of the method proposed in this paper. (c) Comparison of CDF curves of the two methods.

central unsynchronized node has increased slightly compared to the traditional method. It can be seen from Figure 9 that the transmission probability of node 1 is mainly distributed from beam 4 to beam 9, which corresponds to the upper left direction of Figure 7, and the transmit probability of other beams is nearly zero. This is because there are already other synchronized nodes in the beam directions. Therefore, from the perspective of reducing interference and increasing the transmission success rate in other directions, the transmit probability in this direction is reduced.

The simulation is carried out under the same conditions with different numbers of beams. When the number of beams is set to be 10,8 , and 6 , respectively, the CDF curve comparison of the waiting time of the two methods for new nodes is shown in Figure 10. When the number of beams is set to be 10,8 , and 6 , respectively, the synchronization waiting delays of the traditional method with equal transmit probability are 500,320, and 180, respectively. After adopting

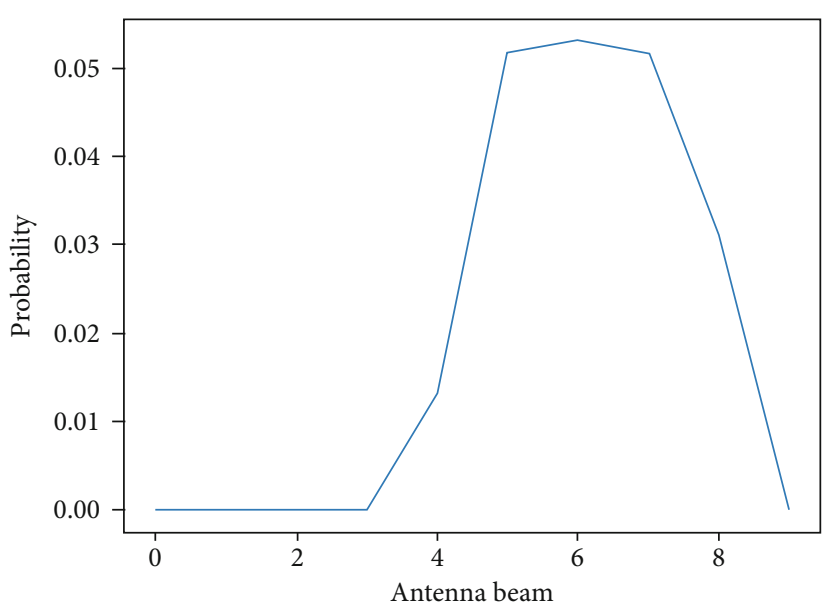

Figure 9: The probability distribution of $K$ beams of node 1. 


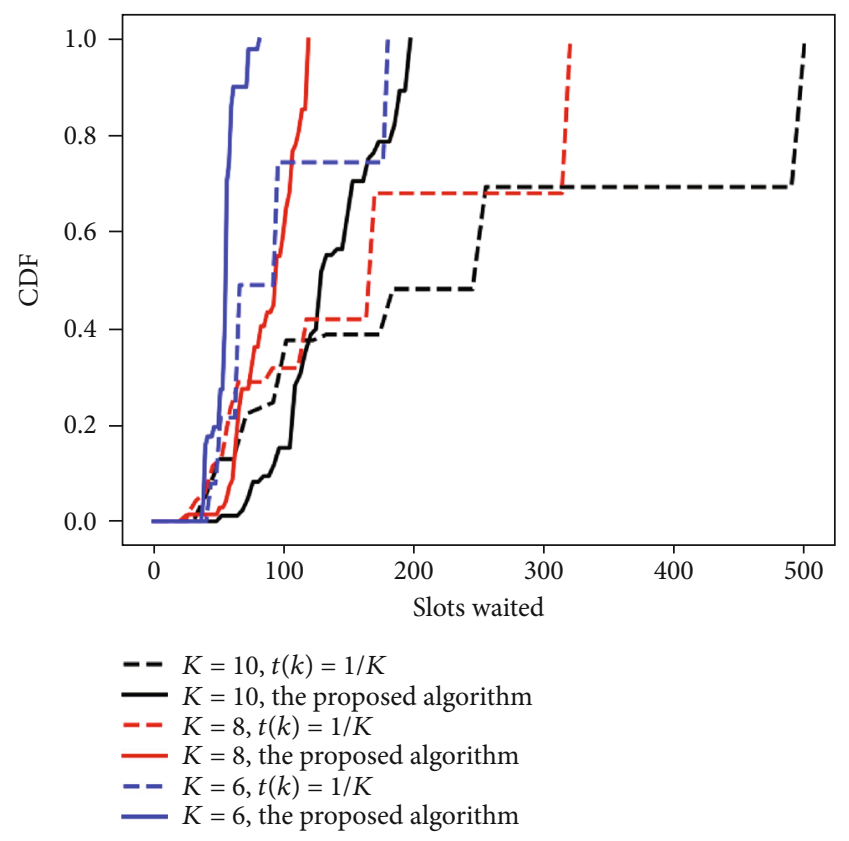

FIgURE 10: Comparison of the CDF curves of the average synchronization waiting delays of the proposed method and traditional method with equal probability of the $K$ beams when the number of beams is set to be 10,8 , and 6 , respectively.

the method proposed in this paper, the synchronization waiting delays are 197, 118, and 81, respectively. Compared with the traditional method, they are reduced by 303,202 , and 99 , respectively, and the reduction ratio is $60.6 \%, 63.1 \%$, and $55 \%$. It can be seen from Figure 10 that compared with the traditional method, the more the beams, the narrower the beam angle; the method proposed in this paper shortens the waiting time of a new node with a larger absolute value. This is because the narrower the beam angle, the smaller the probability of the two-node beams facing each other.

Under the condition of the number of beams $K=10$, when the interference parameters $\Delta$ are set to be 1,2 , and 3 , respectively, the method proposed in this paper is adopted, and the synchronization waiting delays are shown in Figure 11 . When the interference parameters $\Delta$ are set to be 1,2 , and 3 , respectively, under the traditional method with equal transmit probability, the synchronization waiting delay is 500 , and under the proposed method, the synchronization waiting delays are 182, 197 and 206, respectively. Compared with the traditional method, the synchronization waiting delays of the proposed method are reduced by 318,303 , and 294 , respectively, and the reduction ratio is $63.6 \%$, $60.6 \%$, and $58.8 \%$. Figure 11 shows that under the condition of different interference parameters $\Delta$, the largest waiting delay of the traditional method is the same. This is because interference is not the main factor leading to increasing the maximal average self-synchronization time of the unsynchronized node. The main reason for increasing the maximal average self-synchronization time is the unsynchronized node located at the edge of the network, and only one of the synchronized nodes can reach it. At the same time, as shown in Figure 11, as the interference parameter is smaller, the maximum average self-synchronization time of the

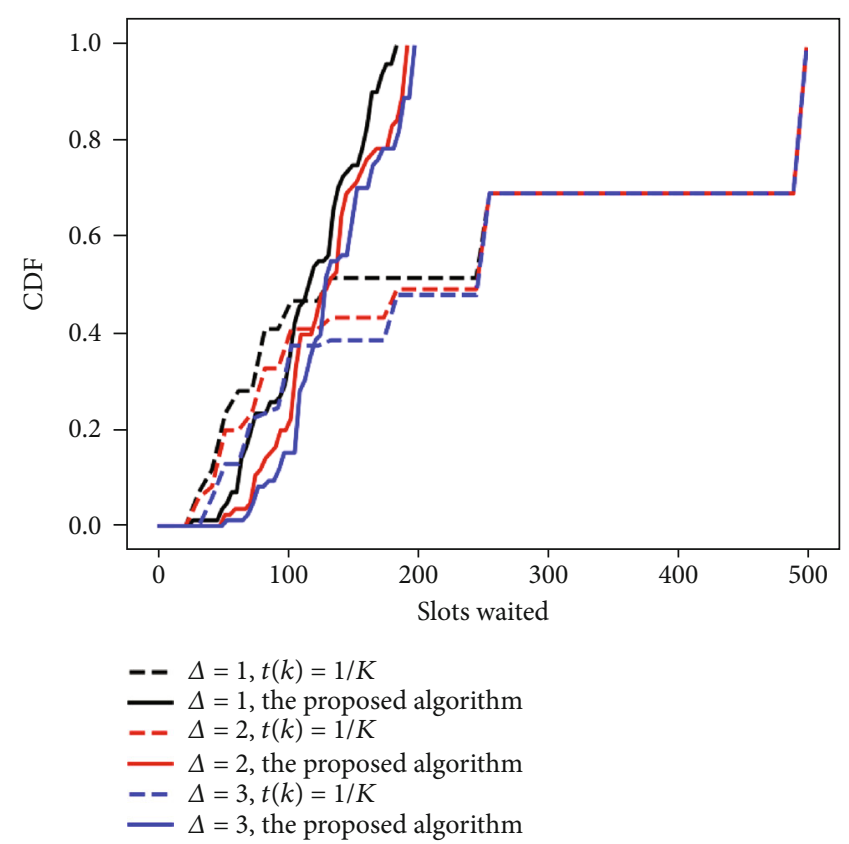

FIgURE 11: Comparison of the CDF curves of the average synchronization waiting delays of the proposed method and traditional method with equal probability of the $K$ beams when the interference parameters $\Delta$ are set to be 1,2 , and 3 , respectively.

method proposed in this paper is smaller. This is because the interference of multiple nodes becomes the main factor restricting the maximal average self-synchronization time after rescheduling the transmit probabilities of different beams using the method proposed in this paper. Therefore, the smaller the interference area, the smaller the maximal average self-synchronization time.

\section{Conclusions}

In this paper, we propose a cooperative antenna selection method for directional antenna ad hoc networks based on ALOHA, which minimizes the maximum average time of self-synchronization of new nodes without external time synchronization and angle synchronization. Firstly, we performed a quantitative analysis of the average self-synchronization time for new nodes in the case of a two-node network, and then we gave the expression for the expected average time of selfsynchronization of new nodes in the case of a multiple-node network. The problem of minimizing the maximum selfsynchronization average time of new nodes is transformed into the problem of finding the minimum value of the infinite norm of the sequence. Based on this, an improved method for cooperating antenna selection of directional antenna ad hoc networks based on ALOHA is proposed. It minimizes the maximum average time of self-synchronization through parameter sharing and relative geometric position relationship and calculates the optimal transmit probability distribution of this node in different directions. Finally, we verify the effectiveness of the method proposed through simulation. In our simulation, the number of beams is set to 10 and the 
interference parameter is set to 2; in the typical scenario of five nodes, the maximum average time of self-synchronization of new nodes is reduced by $60 \%$.

\section{Data Availability}

The data used to support the findings of this study are available from the corresponding author upon request.

\section{Conflicts of Interest}

The authors declare that there is no conflict of interest regarding the publication of this paper.

\section{References}

[1] X. Liu and X. Zhang, "Rate and energy efficiency improvements for 5G-based IoT with simultaneous transfer," IEEE Internet of Things Journal, vol. 6, no. 4, pp. 5971-5980, 2019.

[2] X. Liu and X. Zhang, "NOMA-based resource allocation for cluster-based cognitive industrial Internet of Things," IEEE Transactions on Industrial Informatics, vol. 16, no. 8, pp. 5379-5388, 2020.

[3] X. Liu, X. B. Zhai, W. Lu, and C. Wu, "QoS-guarantee resource allocation for multibeam satellite industrial Internet of Things with NOMA," IEEE Transactions on Industrial Informatics, vol. 17, no. 3, pp. 2052-2061, 2021.

[4] S. Vasudevan, M. Adler, D. Goeckel, and D. Towsley, "Efficient algorithms for neighbor discovery in wireless networks," IEEE/ACM Transactions on Networking, vol. 21, no. 1, pp. 69-83, 2013.

[5] S. Lai, B. Ravindran, and H. Cho, "Heterogenous quorumbased wake-up scheduling in wireless sensor networks," IEEE Transactions on Computers, vol. 59, no. 11, pp. 1562-1575, 2010.

[6] P. Dutta and D. E. Culler, "Practical asynchronous neighbor discovery and rendezvous for mobile sensing applications," in Proceedings of the 6th ACM conference on Embedded network sensor systems - SenSys '08, pp. 71-84, Raleigh, NC, USA, 2008.

[7] A. Kandhalu, K. Lakshmanan, and R. Rajkumar, "U-connect: a low-latency energy-efficient asynchronous neighbor discovery protocol," in Proceedings of the 9th ACM/IEEE International Conference on Information Processing in Sensor Networks IPSN '10, pp. 350-361, Stockholm, Sweden, 2010.

[8] G. H. Hardy and E. M. Wright, An Introduction to the Theory of Numbers, Oxford University Press, New York, 1975.

[9] M. Bakht, M. Trower, and R. H. Kravets, "Searchlight: won't you be my neighbor?," in Proceedings of the 18th annual international conference on Mobile computing and networking Mobicom '12, pp. 185-196, Istanbul, Turkey, 2012.

[10] W. Ye, J. S. Heidemann, and D. Estrin, "An energy-efficient MAC protocol for wireless sensor networks," in Proceedings Twenty-First Annual Joint Conference of the IEEE Computer and Communications Societies, vol. 3, pp. 1567-1576, New York, NY, USA, 2002.

[11] J. Polastre, J. L. Hill, and D. E. Culler, "Versatile low power media access for wireless sensor networks," in Proceedings of the 2nd international conference on Embedded networked sensor systems - SenSys '04, pp. 95-107, Baltimore, MD, USA, 2004.
[12] M. J. McGlynn and S. A. Borbash, "Birthday protocols for low energy deployment and flexible neighbor discovery in ad hoc wireless networks," in Proceedings of the 2nd ACM international symposium on Mobile ad hoc networking of computing - MobiHoc '01, pp. 137-145, Long Beach, CA, USA, 2001.

[13] S. A. Borbash, A. Ephremides, and M. J. McGlynn, "An asynchronous neighbor discovery algorithm for wireless sensor networks," Ad Hoc Networks, vol. 5, no. 7, pp. 998-1016, 2007.

[14] M. J. McGlynn and S. A. Borbash, "Birthday protocols for low energy deployment and flexible neighbor discovery ad hoc wireless networks," in Proceedings of the 2nd ACM international symposium on Mobile ad hoc networking \& computing - MobiHoc '01, pp. 137-145, Long Beach, CA, USA, 2001.

[15] S. Vasudevan, J. F. Kurose, and D. F. Towsley, "On neighbor discovery wireless networks with directional antennas," in Proceedings IEEE 24th Annual Joint Conference of the IEEE Computer and Communications Societies, vol. 4, pp. 2502-2512, Miami, FL, USA, 2005.

[16] A. Russell, S. Vasudevan, B. Wang, W. Zeng, X. Chen, and W. Wei, "Neighbor discovery in wireless networks with multipacket reception," IEEE Transactions on Parallel and Distributed Systems, vol. 26, no. 7, pp. 1984-1998, 2015.

[17] X. An and R. Hekmat, "Self-adaptive neighbor discovery in ad hoc networks with directional antennas," in 2007 16th IST Mobile and Wireless Communications Summit, pp. 1-5, Budapest, Hungary, 2007.

[18] D. Ji, Z. Wei, X. Chen et al., "Radar-communication integrated neighbor discovery for wireless ad hoc networks," in 2019 11th International Conference on Wireless Communications and Signal Processing (WCSP), pp. 1-5, Xi'an, China, 2019.

[19] G. Pei, M. M. Albuquerque, J. H. Kim, D. P. Nast, and P. R. Norris, "A neighbor discovery protocol for directional antenna networks," in MILCOM 2005 - 2005 IEEE Military Communications Conference, vol. 1, pp. 487-492, Atlantic City, NJ, USA, 2005.

[20] R. Ramanathan, J. Redi, C. Santivanez, D. Wiggins, and S. Polit, "Ad hoc networking with directional antennas: a complete system solution," in 2004 IEEE Wireless Communications and Networking Conference (IEEE Cat. No.04TH8733), vol. 1, pp. 375-380, Atlanta, GA, USA, 2005.

[21] W. Xiong, B. Liu, and L. Gui, "Neighbor discovery with directional antennas in mobile ad-hoc networks," in 2011 IEEE Global Telecommunications Conference - GLOBECOM 2011, pp. 1-5, Houston, TX, USA, 2011.

[22] H. Cai and T. Wolf, "On 2-way neighbor discovery in wireless networks with directional antennas," in 2015 IEEE Conference on Computer Communications (INFOCOM), pp. 702-710, Hong Kong, China, 2015.

[23] Joon-Sang Park, Sung-Woo Cho, M. Y. Sanadidi, and M. Gerla, "An analytical framework for neighbor discovery strategies in ad hoc networks with sectorized antennas," IEEE Communications Letters, vol. 13, no. 11, pp. 832-834, 2009.

[24] Z. Zhang, "Pure directional transmission and reception algorithms in wireless ad hoc networks with directional antennas," in IEEE International Conference on Communications, pp. 3386-3390, Seoul, South Korea, 2005.

[25] K. F. Hasan, C. Wang, Y. Feng, and Y. C. Tian, "Time synchronization in vehicular ad-hoc networks: a survey on theory and practice," Vehicular Communications, vol. 14, pp. 39-51, 2018. 
[26] D. L. Mills, "Internet time synchronization: the network time protocol," IEEE Transactions on Communications, vol. 39, no. 10, pp. 1482-1493, 1991.

[27] J. van Greunen and J. Rabaey, "Lightweight time synchronization for sensor networks," in Proceedings of the 2Nd ACM International Conference on Wireless Sensor Networks and Applications, WSNA '03, pp. 11-19, San Diego, CA, USA, 2003.

[28] S. Ganeriwal, R. Kumar, and M. B. Srivastava, "Timing-sync protocol for sensor networks," in Proceedings of the 1st International Conference on Embedded Networked Sensor Systems, SenSys '03, pp. 138-149, Los Angeles, California, USA, 2003.

[29] M. Maróti, B. Kusy, G. Simon, and A. Lédeczi, “The flooding time synchronization protocol," in Proceedings of the 2nd international conference on Embedded networked sensor systems, pp. 39-49, Baltimore, MD, USA, 2004.

[30] F. Baccelli, B. Błaszczyszyn, M.-O. Haji-Mirsadeghi, and M. Omid, "Optimal paths on the space-time SINR random graph," Advances in Applied Probability, vol. 43, no. 1, pp. 131-150, 2011.

[31] J. D. Hedengren, R. Asgharzadeh Shishavan, K. M. Powell, and T. F. Edgar, "Nonlinear modeling, estimation and predictive control in APMonitor," Computers and Chemical Engineering, vol. 70, pp. 133-148, 2014.

[32] L. D. R. Beal, D. Hill, R. A. Martin, and J. D. Hedengren, "GEKKO optimization suite," Processes, vol. 6, no. 8, 2018. 